\title{
Towards Spectral-Texture Approach to Hyperspectral Image Analysis for Plant Classification
}

\author{
Document Version \\ Accepted author manuscript
}

Link to publication record in Manchester Research Explorer

\section{Citation for published version (APA):}

AlSuwaidi, A., Grieve, B., \& Yin, H. (2017). Towards Spectral-Texture Approach to Hyperspectral Image Analysis for Plant Classification. In H. Yin, Y. Gao, S. Chen, Y. Wen, G. Cai, T. Gu, J. Du, A. J. Tallón-Ballesteros, \& M. Zhang (Eds.), Intelligent Data Engineering and Automated Learning -- IDEAL 2017 (pp. 251-260). (Lecture Notes in Computer Science; Vol. 10585). Springer Nature. https://link.springer.com/chapter/10.1007\%2F978-3-31968935-7_28

\section{Published in:}

Intelligent Data Engineering and Automated Learning -- IDEAL 2017

\section{Citing this paper}

Please note that where the full-text provided on Manchester Research Explorer is the Author Accepted Manuscript or Proof version this may differ from the final Published version. If citing, it is advised that you check and use the publisher's definitive version.

\section{General rights}

Copyright and moral rights for the publications made accessible in the Research Explorer are retained by the authors and/or other copyright owners and it is a condition of accessing publications that users recognise and abide by the legal requirements associated with these rights.

\section{Takedown policy}

If you believe that this document breaches copyright please refer to the University of Manchester's Takedown Procedures [http://man.ac.uk/04Y6Bo] or contact uml.scholarlycommunications@manchester.ac.uk providing relevant details, so we can investigate your claim.

\section{OPEN ACCESS}




\title{
Toward Spectral-Texture HSI Analysis Tool for Plant Classification
}

\author{
Ali AlSuwaidi, Bruce Grieve, and Hujun Yin \\ School of Electrical and Electronic Engineering, The University of Manchester, \\ Manchester, M13 9PL, UK \\ ali.bghalsuwaidi@postgrad.manchester.ac.uk, \\ bruce.grieve@manchester.ac.uk, hujun.yin@manchester.ac.uk
}

\begin{abstract}
The popularity of using hyperspectral imaging systems in studying plant properties, types, and condition has increased due to the numerous economical and financial advantages of such systems. This paper is concerned with the analysis of different plant conditions using proximal hyperspectral imaging systems. A spectral-texture approach based on correlation between feature selection and the Markov random field model is introduced to enhance prediction performance, as compared to the use of the spectral and texture analysis approaches individually. Two independent hyperspectral datasets captured by two proximal hyperspectral instrumentations, with different acquisition dates and exposure times, were used in the evaluation. Experimental results show promising improvement in the discrimination performance of the presented approach. The study shows that such an approach can shed light on attributes that better differentiate plants, their properties, and conditions.
\end{abstract}

Keywords: Feature selection . Hyperspectral imaging . Markov random field. Spectral analysis. Texture analysis.

\section{Introduction}

Since its discovery in the early 1980s, imaging spectrometry has attracted a considerable amount of interest in innovative scientific quests due to its ability to sense a wider range of electromagnetic spectrum [12]. Hyper-spectral imaging (HSI), a branch of multivariate imaging [10], gathers optical properties of the target with several spectral representations using a mixture of spectroscopy and remote imaging technologies [7]. HSI has been utilised in several applications; for instance, remote sensing [5], proximal sensing [15], industrial processes [6], medical imaging [18], and chemical processes [9]. Moreover, several configurations have been used to capture hyperspectral images [21] point, line, area, and single shot scanning.

Texture and spectral information are the fundamental properties of hyperspectral images. Texture information is described as an attribute representing the texture arrangement of grey levels [3]; minimum and maximum values represent 
the darkest and brightest points respectively through an image. This information is associated with many texture properties such as coarseness, smoothness, orientation, depth, etc. It should be mentioned that pixels (i.e. texture unit element) are used to represent single or several texture features. Meanwhile, the spectral information defines the entire measured spectrum of the corresponding texture, where each pixel in the image is represented by a unique spectral signature [7]. It is worth noting that the spectrum information might cover single or several parts of the electromagnetic spectrum.

Several texture and spectral analysis techniques have been introduced to analyse hyperspectral images. These techniques have played an important role in several domains such as agriculture, medicine, and industry for many tasks - especially image classification [3]. Since hyperspectral imaging senses a wider range of the electromagnetic spectrum, effective and efficient approaches are needed before analysing the images [1]. These approaches include feature extraction and feature selection, used to reduce the dimensionality of hyperspectral images as well as the requested processing time, thus analysing only the information relevant to the investigated problem.

Texture analysis gives insight about the texture, thus providing an important base to object recognition and description. Generally, texture analysis techniques can be broadly categorised into statistical, structural, transform-based, and model-based [3]. The first two use the statistics, i.e. distribution of the grey levels, and the arrangement rules of the texture respectively to describe the texture. Moreover, the characteristics of the transformed texture are used to describe textures in the transform-based technique, while the model-based approach uses estimated model parameters. Several studies published in the past have shown the Markov random field (MRF) to be one of the most powerful models to describe different textures [22]. MRF, i.e. inter-pixel dependency, has been utilised in different applications such as image denoising, image compression, image segmentation, super-resolution, etc.

This paper focuses on classifying different plant conditions (i.e. stressed vs. normal; diseased vs. healthy) using the spectral-texture approach and compares the results with those from using individual spectral or texture approaches. The relevant features (i.e. wavelengths in this experiment) along with the texture representation (i.e. estimated MRF parameters) will be used in the classification stage. Furthermore, a conventional support vector machine (SVM) was used for two reasons [2]: 1) it is considered as a state of the art classification algorithm, 2 ) it reduces the risk of overfitting (i.e. in order to deal efficiently with the dimensionality problem). The classification result was promising and an improved discrimination can be achieved.

The remainder of the paper is organised as follows: the background is reviewed in Sect. 2. Section 3 presents HSI systems, HSI datasets, and the spectraltexture approach. Results and their discussion are given in Sect. 4, followed by the concluding remarks in section Sect. 5. 


\section{Background}

This section is divided into two subsections. An overview of feature selection is presented in the first subsection, while the Markov random field (MRF) model is highlighted in the second.

\section{$2.1 \quad$ Feature Selection}

HSI systems gather large amounts of information; however, not all the data collected is necessarily relevant to the problem investigated. The problem of high dimensionality can be alleviated by using a feature selection process. Feature selection is the process of choosing a relevant subset of features (in this study, wavelengths) and discarding the remaining ones (e.g. irrelevant and redundant) $[16,17]$. The process of feature selection can be described in four steps: search organisation, subset evaluation, stopping criteria, and result validation. The first step is responsible for generating several subsets of features and that includes determining search direction and procedure. The second step involves evaluation of the relevance of the generated subsets, based on certain criteria, in order to select the optimal one (i.e. the one that maximises the evaluation criteria). The last two steps determine when the process should be halted and the significance of the selection parameters to the investigated problem.

Feature selection models can be separated - based on certain evaluation criteria - into the following categories: filter, wrapper, and embedded [16, 17]. The discrimination capability depends solely on data characteristics in the first model, while it depends on the mining algorithms used to assess the relevancy of the features. It should be noted that the embedded model was introduced to utilise both filter and wrapper models, i.e. to rank features based on their data characteristics and evaluate their goodness through classification algorithms. In addition, the filter model can produce acceptable to good performances in short time, while the wrapper and embedded models are easy to implement.

Various feature selection algorithms have been introduced in the past. The correlation-based feature selection (CFS) [13] algorithm has been shown to be particularly powerful due to its ability to discard irrelevant and redundant features, as well as producing good discrimination performance compared to other selection algorithms [2]. It uses Shannon's entropy $H(\boldsymbol{x})=-\sum_{i=1}^{n} P\left(x_{i}\right) \log _{2} P\left(x_{i}\right)$ and information gain $I(\boldsymbol{x}, \boldsymbol{y})=H(\boldsymbol{x})-H(\boldsymbol{x} \mid \boldsymbol{y})$ to minimise feature bias and then measures the correlation between the features and the classes. The measured correlation is then used to evaluate the feature heuristically:

$$
\operatorname{Merit}_{S}=\frac{n\left(\overline{r_{c f}}\right)}{n+\left(n+n(n-1) \overline{r_{f f}}\right)}
$$

where $\overline{r_{c f}}$ denotes the average feature-class correlation, $\overline{r_{f f}}$ represents the average feature-feature correlation, and Merit $_{S}$ is the heuristic merit of a subset containing features. 


\subsection{MRF Model}

An MRF, a model-based texture, is an extension of the Markov chain model [4]. It has a set of nodes, each of which corresponds to a variable or set of variables (an example of MRF neighbour structure and equivalent parameters is shown in Fig. 1). It is also termed an undirected graph model since it is more natural for modeling certain problems, such as spatial statistics and image analysis [20]. Moreover, the orientation of the texture features is not required, unlike the directed graph model. The main advantages of MRF models compared to directed graph models are: 1) more natural for certain domains (i.e. symmetric) and 2) the discrimination of former models work better than the latter one due to the normalisation process (i.e. globally vs. locally). In contrast, the major disadvantages are: 1) less interoperable and 2) parameter estimation can be computationally more expensive (e.g. maximum likelihood estimate).
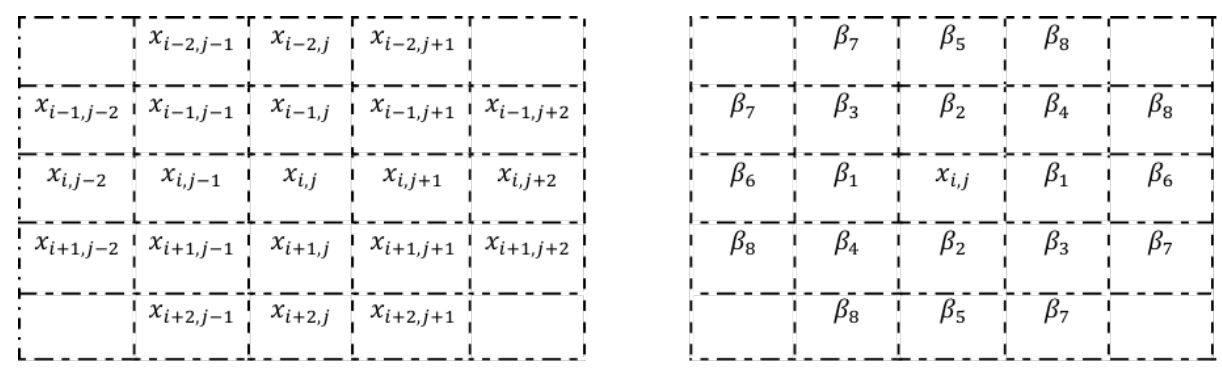

Fig. 1: Markov random field. Neighbour structure (left) and equivalent parameters of $x_{i, j}$ (right).

The MRF can be mathematically described using the equivalent Gibbs distribution with regard to the same graph [11]. Let $P(x)$ denotes a Gibbs distribution for realisation $x, \mathcal{N}$ represents a neighbouring system, $\Omega$ denotes a finite lattice, $\mathcal{C}$ represents all possible cliques, i.e. the subset of a lattice consists of single and/or set of pixels which are neighbours to each other, then the distribution can be represented as:

$$
P(x)=\frac{1}{Z} e^{-U(x) / T}
$$

where $T$ is a constant and stands for temperature; $U(x)$ represents the energy function that depends only on clique potential $V_{C}$ on the lattice and can be written as:

$$
U(x)=\sum_{c \in \mathcal{C}} V_{c}(x)
$$

$Z$ denotes a normalising constant, also termed as partition function, and defined as:

$$
Z=\sum_{x} e^{-U(x) / T}
$$


In terms of estimation of texture parameters, least square (LS) and maximum likelihood (ML) are two estimates that are widely used with the texture [22]. The former is very simple and it has low computational requirements compared to the latter, which is why it is more preferable in the estimation process. For the LS estimate, the parameters over a finite lattice $\Omega$ can be estimated using the following equation:

$$
\hat{\beta}=\left[\sum_{m \in \Omega} \beta_{m} \beta_{m}^{T}\right]^{-1}\left[\sum_{m \in \Omega} \sum_{i, j \in \Omega} \beta_{m} x_{i, j}\right], m=1,2, \ldots, M
$$

where $x_{i, j}$ represents the middle pixel and $\beta_{m}$ denotes the neighbouring pixels that can be represented as:

$$
\beta_{m}=\operatorname{col}\left[x_{i+u, j+v}\right],(u, v) \neq 0,(u, v) \in \mathcal{N}
$$

where $u, v$ represent the location of the neighbouring pixels horizontally and vertically respectively and col stands for column. It is worth noting that LS is not consistent for non-causal neighbour sets [20,22]. However, it is more preferable compared to the ML estimate since it is computationally expensive. In addition, the ML result is not always guaranteed (if not impossible) and requires an alternative function, i.e. iterative and computationally expensive, such as pseudo likelihood (MPL).

\section{Materials and Methods}

This section describes the materials and the spectral-texture approach. It first emphasises the specifications of the HSI systems used to capture the hyperspectral datasets and then describes the datasets used in the experiment, followed by the description of the spectral-texture approach.

\subsection{HSI Systems and Datasets}

Two HSI systems were used to collect the hyperspectral images: The University of Manchester (UoM) HSI system and The University of Bonn (Bonn) HSI system. The key specifications of both systems are given in Table 1. Both systems operate in controlled environments (dark room vs. dark chamber) in order to minimize the effect of unwanted noise. Furthermore, the dynamic range of both systems is managed to prevent saturation. In addition, three images (scene, dark noise, and flat field) are captured by both systems and then used to spectrally normalise the scene images to enhance the quality of the image. More information about both systems can be found in [8] and [19].

Two HSI datasets (called UoM and Bonn for simplicity) captured with different acquisition dates and exposure times were used for analysis purposes. The scene images of the UoM dataset consisted of six Arabidopsis leaf samples, while the Bonn dataset consisted of four sugar leaf samples [19] placed flattened on the sample plate in both cases (shown in Fig. 2). Moreover, the former dataset 
Table 1: Key specifications of UoM and Bonn HSI systems

\begin{tabular}{l|l|l}
\hline Specification & HSI Systems & \\
\cline { 2 - 3 } & UoM & Bonn \\
\hline Sensor type & Area & Line \\
Effective Pixels & $1024 \times 1344$ & 1600 \\
Spectral range & $400-700 \mathrm{~nm}$ & $400-1000 \mathrm{~nm}$ \\
Spectral resolution & $10 \mathrm{~nm}$ & $2.8 \mathrm{~nm}$ \\
Spatial resolution & High & $0.19 \mathrm{~mm}$ \\
Radiometric resolution & 12 -bits & 12 -bits \\
Dispersion device & liquid crystal tuneable filter & Image Spectrograph \\
\hline
\end{tabular}

consists of two normal and four stressed (cold and heat) leaves (i.e. top and bottom left: normal, middle top and bottom: cold stress, and top and bottom right: heat stress), while the latter consists of four leaves under one condition; either healthy (controlled) or unhealthy (Cercospora). 648 samples were extracted from the UoM dataset and divided into two groups: normal and stressed. The normal group was represented by 216 samples and the remaining represented stressed samples. The Bonn dataset yielded 196 samples: 98 samples of controlled and Cercospora conditions. It should be stated that only green areas of both datasets were considered for sample extraction. In addition, $50 \%$ of the samples were used for training purposes with 10-fold cross validation with the remaining ones used for testing.

\section{$3.2 \quad$ Spectral-Texture Approach}

Our spectral-texture approach (illustrated in Fig. 3) can be described in four steps: spectral signature extraction, significant wavelengths selection, texture parameters estimation, and classification. The spectral signature was extracted from the pixel value of the small leaf region and then averaged over the entire wavelengths spectrum. This averaged signature was used to reduce the variation in pixel intensities across the selected leaf region. CFS was used in the wavelengths selection step in order to simplify the dataset and select the most significant wavelengths. The LS estimate was used to estimate the second order parameters of the MRF model and then average them either over the entire wavelength spectrum or over the list of selected wavelengths. In the final step, the selected wavelength and the estimated texture parameters were combined and passed to a conventional SVM classifier with a radial basis function (RBF) kernel for classification. The SVM uses a quadratic programming routine to solve the following quadratic problem with the regards to training set in order to find the best hyperplane [14]: 


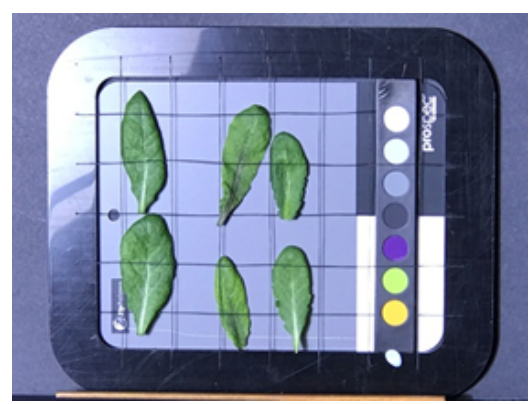

(a)

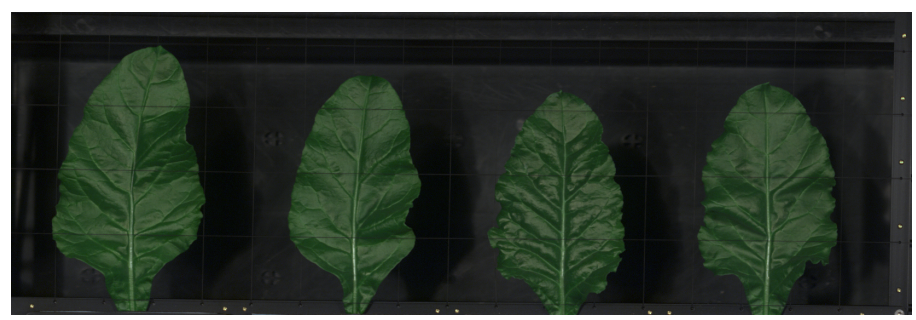

(b)

Fig. 2: Samples of UoM and Bonn scene images. (a) UoM Arabidopsis samples captured on February 2017(b) Bonn Sugar samples captured on March 2013.

$$
\begin{gathered}
\min _{\omega \in \mathbb{R}^{d}, \xi_{i} \in \mathbb{R}^{+}}\|\omega\|^{2}+C \sum_{i}^{N} \xi_{i} \\
\text { subject to }: y_{i}\left(\omega \cdot \boldsymbol{x}_{\boldsymbol{i}}+b\right) \geq 1-\xi_{i}
\end{gathered}
$$

where $\omega, b, \boldsymbol{x}_{\boldsymbol{i}}, y_{i}, \xi_{i}$ represent the weight vector, bias, training set, desired class label, and a non-zero slack variable respectively. Moreover, $C$ is the regularisation parameter and it is used to mark the misclassified samples, thus determining the flexibility of the decision boundary. In this case, the decision function $y$ can be solved using the weight vector as well as the bias:

$$
y=\operatorname{sign}\left(\omega \cdot \boldsymbol{x}_{\boldsymbol{i}}+b\right)
$$

The value of decision function $y \in\{ \pm 1\}$, where 1 denotes one class and -1 the other class. It should be mentioned that false positive and negative errors have to be reduced in order to obtain a good classification result. In addition, the RBF kernel was used to employ the nonlinear hyperplane and can be defined as the following exponential function:

$$
K(\boldsymbol{x}, \boldsymbol{y})=e^{-\gamma\|\boldsymbol{x}-\boldsymbol{y}\|^{2}}, \gamma=\frac{1}{2 \sigma^{2}}
$$




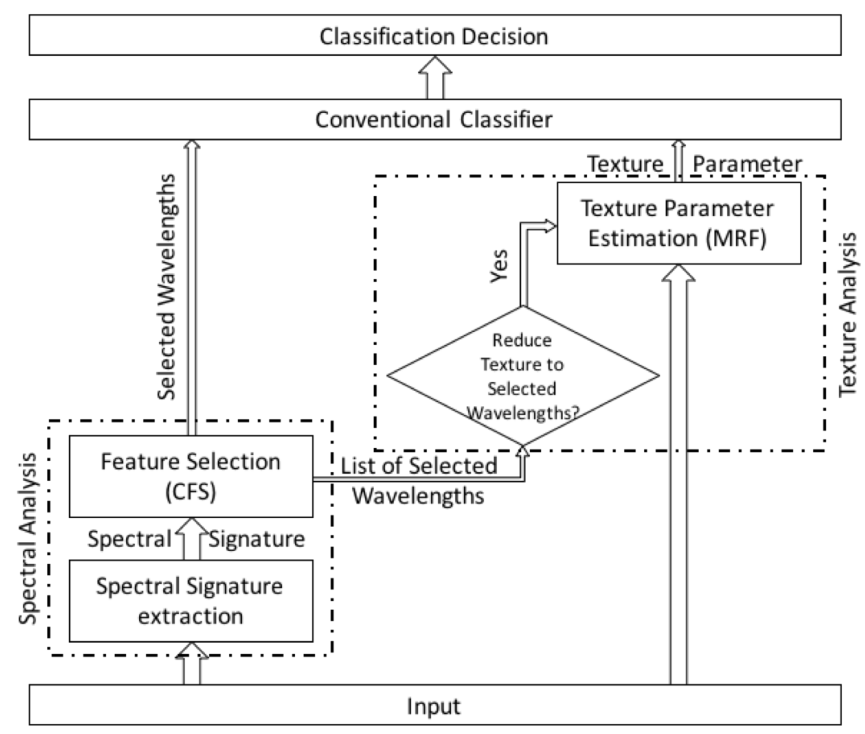

Fig. 3: Schematic of spectral-texture analysis approach

\section{Results and Discussions}

The experimental results assessed the usefulness of the spectral-texture approach in analysing and classifying plant hyperspectral images under different conditions. Both UoM and Bonn datasets were used in the analysis. The final results were then compared with the classification results of existing spectral analysis approach, texture analysis approach, and the combination of all the wavelengths and the estimated texture parameters. $50 \%$ of the samples were used for training with 10-fold cross validation and the other $50 \%$ samples were used for testing. Table 2 displays the average classification of 100 run with the standard deviation.

Table 2: Average classification rate

\begin{tabular}{l|l|l}
\hline \multirow{2}{*}{ Technique } & \multicolumn{2}{|l}{$\begin{array}{l}\text { Average classification rate (\%) } \\
\text { (standard deveiation) }\end{array}$} \\
\cline { 2 - 3 } & UoM & Bonn \\
\hline All wavelengths & $87.35(0.019)$ & $98.54(0.019)$ \\
CFS & $88.40(0.014)$ & $98.85(0.010)$ \\
MRF & $74.64(0.019)$ & $65.42(0.037)$ \\
All wavelengths + MRF & $83.42(0.020)$ & $88.51(0.042)$ \\
CFS + MRF & $\mathbf{9 2 . 8 7}(\mathbf{0 . 0 0 9})$ & $\mathbf{9 9 . 3 6}(\mathbf{0 . 0 0 7})$ \\
\hline
\end{tabular}


What stands out in this table is that the average classification rate of the spectral-texture approach outperforms other approaches, including the combination of all the wavelengths and the estimated texture parameters (percentage respectively). The results suggest this is a valid approach for studying and analysing different plant types and plant condition. Further statistical tests revealed that the improvements are significant compared to the other approaches at a significance level of $1 \%$, especially the wavelengths-texture and texture approaches $\left(\mathrm{p}\right.$-value $\left.<10^{-5}\right)$.

\section{Conclusions}

This paper presented a spectral-texture approach for analysis and classification of hyperspectral plant types and condition. The experimental results from this approach have shown an improvement in discrimination performance compared to other approaches. Moreover, the significance of the spectral-texture approach at $1 \%$ significance level was demonstrated through a statistical test. The findings suggest that such an approach seems valid and applicable for the study of different plant properties, types, and conditions. Future study can explore the effect of the estimated parameters with different orders (e.g. first and third orders MRF), as well as different classification routines, such as novelty detection to best identify plant properties, types, and conditions.

\section{References}

1. AlSuwaidi, A., Veys, C., Hussey, M., Grieve, B., Yin, H.: Hyperspectral feature selection ensemble for plant classification. In: Hyperspectral Imaging and Applications (HSI 2016) (Oct 2016)

2. AlSuwaidi, A., Veys, C., Hussey, M., Grieve, B., Yin, H.: Hyperspectral selection based algorithm for plant classification. In: 2016 IEEE International Conference on Imaging Systems and Techniques (IST). pp. 395-400 (Oct 2016)

3. Bharati, M.H., Liu, J., MacGregor, J.F.: Image texture analysis: methods and comparisons. Chemometrics and Intelligent Laboratory Systems 72(1), $57-71$ (2004)

4. Blake, A., Kohli, P., Rother, C.: Markov Random Fields for Vision and Image Processing. The MIT Press (2011)

5. Campbell, J., Wynne, R.: Introduction to Remote Sensing. Guilford Publications, fifth edn. (2011)

6. Duchesne, C., Liu, J., MacGregor, J.: Multivariate image analysis in the process industries: A review. Chemometrics and Intelligent Laboratory Systems 117, 116 - 128 (2012)

7. ElMasry, G., Sun, D.W.: Chapter 1 - principles of hyperspectral imaging technology. In: Sun, D.W. (ed.) Hyperspectral Imaging for Food Quality Analysis and Control, pp. 3 - 43. Academic Press, San Diego (2010)

8. Foster, D.H., Amano, K., Nascimento, S.M.C.: Color constancy in natural scenes explained by global image statistics. Visual Neuroscience 23(3-4), 341349 (2006) 
9. Geladi, P., Bengtsson, E., Esbensen, K., Grahn, H.: Image analysis in chemistry i. properties of images, greylevel operations, the multivariate image. TrAC Trends in Analytical Chemistry 11(1), 41 - 53 (1992)

10. Geladi, P.L.M., Grahn, H.F., Burger, J.E.: Multivariate images, hyperspectral imaging: Background and equipment. In: Techniques and Applications of Hyperspectral Image Analysis, pp. 1-15. John Wiley and Sons, Ltd (2007)

11. Geman, S., Geman, D.: Stochastic relaxation, gibbs distributions, and the bayesian restoration of images. IEEE Transactions on Pattern Analysis and Machine Intelligence PAMI-6(6), 721-741 (Nov 1984)

12. Goetz, A.F., Vane, G., Solomon, J.E., Rock, B.N.: Imaging spectrometry for earth remote sensing. Science 228(4704), 1147-1153 (1985)

13. Hall, M.A., Smith, L.A.: Feature selection for machine learning: Comparing a correlation-based filter approach to the wrapper. In: Proceedings of the Twelfth International Florida Artificial Intelligence Research Society Conference. pp. 235-239 (1999)

14. Kulkarni, S., Harman, G.: An Elementary Introduction to Statistical Learning Theory. Wiley Publishing, 1st edn. (2011)

15. Liu, H., Lee, S.H., Chahl, J.S.: Development of a proximal machine vision system for off-season weed mapping in broadacre no-tillage fallows. Journal of Computer Science 9(12), 1803-1821 (213)

16. Liu, H., Motoda, H.: Feature Selection for Knowledge Discovery and Data Mining. Kluwer Academic Publishers, Norwell, MA, USA (1998)

17. Liu, H., Yu, L.: Toward integrating feature selection algorithms for classification and clustering. IEEE Transactions on Knowledge and Data Engineering 17(4), 491-502 (April 2005)

18. Lu, G., Fei, B.: Medical hyperspectral imaging: a review. Journal of Biomedical Optics 19(1), 010901 (2014)

19. Mahlein, A.K., Hammersley, S., Oerke, E.C., Dehne, H.W., Goldbach, H., Grieve, B.: Supplemental blue led lighting array to improve the signal quality in hyperspectral imaging of plants. Sensors 15(6), 12834-12840 (2015)

20. Murphy, K.P.: Machine Learning: A Probabilistic Perspective. The MIT Press (2012)

21. Qin, J.: Chapter 5 - hyperspectral imaging instruments. In: Sun, D.W. (ed.) Hyperspectral Imaging for Food Quality Analysis and Control, pp. 129 - 172. Academic Press, San Diego (2010)

22. Yin, H., Allinson, N.M.: Self-organised parameter estimation and segmentation of mrf model-based texture images. In: Image Processing, 1994. Proceedings. ICIP94., IEEE International Conference. vol. 2, pp. 645-649. IEEE (1994) 